\title{
Halide Perovskite 3D Photonic Crystals for Distributed Feedback Lasers
}

\author{
Stefan Schünemann, ${ }^{\dagger}$ Sarah Brittman, ${ }^{\ddagger}$ Kun Chen, ${ }^{\dagger \oplus}$ Erik C. Garnett, ${ }^{\ddagger}$ and Harun Tüysüz ${ }^{*},{ }^{\dagger}$ \\ ${ }^{\dagger}$ Max-Planck-Institut für Kohlenforschung, Kaiser-Wilhelm-Platz 1, 45470 Mülheim an der Ruhr, Germany \\ "Center for Nanophotonics, AMOLF, Science Park 104, Amsterdam 1098 XG, The Netherlands
}

Supporting Information

ABSTRACT: Halide perovskites are under intense investigation for light harvesting applications in solar cells. Their outstanding optoelectronic properties such as long charge carrier diffusion lengths, high absorption coefficients, and defect tolerance also has triggered interest in laser and LED applications. Here, we report on the lasing properties of 3D distributed feedback halide perovskite nanostructures prepared via an all-solution process. A colloidal crystal templating approach was developed to precisely control the hybrid halide perovskite structure on the nanoscale. The prepared $\mathrm{CH}_{3} \mathrm{NH}_{3} \mathrm{PbBr}_{3}$ thin films with inverse opal morphology
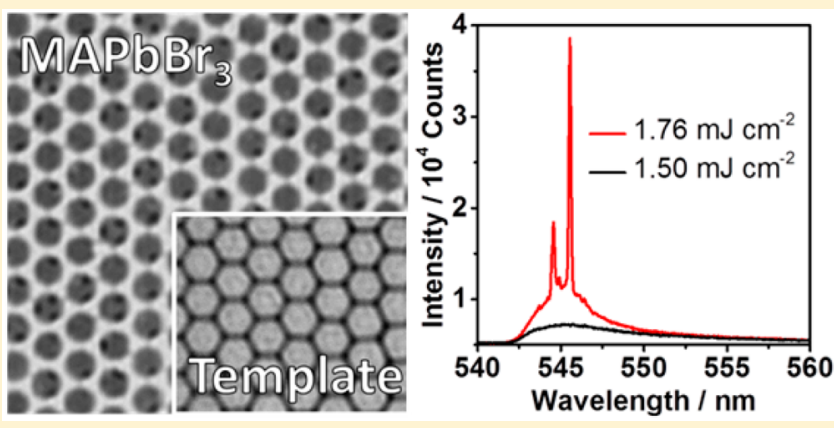
show narrow lasing emissions with a full width half-maximum as low as $0.15 \mathrm{~nm}$ and good long-term stability under pulsed laser excitation above the lasing threshold of $1.6 \mathrm{~mJ} \mathrm{~cm}^{-2}$ in ambient atmosphere. Furthermore, lasing emission was also observed for $\mathrm{CH}_{3} \mathrm{NH}_{3} \mathrm{PbI}_{3}$ inverse opals under excitation with a focused laser beam. Unlike other protocols for the fabrication of distributed feedback perovskite lasers, control of the nanostructure of hybrid halide perovskites is achieved without the use of expensive and elaborate lithography techniques or high temperatures. Therefore, the presented protocol opens a route to the low cost fabrication of hybrid halide perovskite lasers.

KEYWORDS: lead halide perovskites, laser, inverse opal, distributed feedback

ver the past years, halide perovskites have emerged as an attractive material class for diverse optoelectronic applications. Their versatility in optoelectronics arises from several beneficial properties such as long carrier lifetimes, ${ }^{1}$ high absorption coefficients at energies above the bandgap, ${ }^{2}$ and their crystallization at room temperature, which allows inexpensive solution-based syntheses of optoelectronic devices at or near room temperature. ${ }^{3-5}$

Even though halide perovskites have been known since $1893,{ }^{6}$ initial research on their electronic properties by Mitzi and co-workers started only in the 1990s. ${ }^{7,8}$ The research interest in the optoelectronic properties of halide perovskites started with the first report by Miyasaka et al. about a halide perovskite sensitized solar cell in 2009. 9 Since then, accompanied by structural changes, halide perovskite solar cells showed rapidly increasing efficiencies. ${ }^{10}$ The beneficial optoelectronic properties of halide perovskites also led to the development of light emitting diodes and, more recently, lasers. $^{11-19}$

Lasing was firstly demonstrated from optically pumped cavity lasers, ${ }^{20}$ single crystals, ${ }^{21}$ nanodisks, ${ }^{22,23}$ nanowires, ${ }^{18,24,25}$ and nanoplatelets ${ }^{17}$ fabricated by chemical vapor deposition or solution based processes. More recently, lasing from $1 \mathrm{D}$ and 2D halide perovskite distributed feedback (DFB) structures was reported. ${ }^{15,16,26,27}$ Furthermore, Grätzel and co-workers achieved lasing by infiltrating the naturally occurring photonic crystal formed by butterfly wings. ${ }^{28}$ Unlike conventional laser structures, in which the gain medium is embedded in a resonator structure (usually two mirrors) to provide optical feedback, in a DFB structure the feedback mechanism is distributed over the gain medium. ${ }^{29}$ This is usually achieved via a periodic refractive index change, which results in a photonic band structure in which stop-gaps open up resulting in anisotropic propagation of light within the gain medium. The center wavelength of the photonic bandgap $\lambda_{\max }$ can be calculated from the modified Bragg law (eq 1) and depends on the lattice parameter $d$, the filling fractions $f$ and the refractive indices $n$ of the two media, and the angle of the incident light $\theta .^{30}$

$$
\lambda_{\max }=2 d \sqrt{\left(f_{1} n_{1}+\left(1-f_{1}\right) n_{2}\right)^{2}-\sin ^{2}(\theta)}
$$

Optically pumped lasing from 1D and 2D DFB structures has already been demonstrated for halide perovskite materials. In these $1 \mathrm{D}$ and 2D DFB structures, the optical feedback is provided by patterned substrates, which are covered by a halide perovskite layer. ${ }^{15,16,27}$ To obtain spatially well-defined feedback structures, the patterned substrates are prepared via costintense and elaborate lithography techniques. ${ }^{15,16,26,27}$ In these

Received: July 17, 2017

Published: September 7, 2017 
reported structures, the refractive index contrast is formed between the patterned substrate $\left(\mathrm{SiO}_{2}, \mathrm{Si}, \mathrm{Au}\right.$, organic polymers, and natural butterfly wing) and the halide perovskite material. However, to achieve a higher refractive index contrast, it is favorable to prepare a DFB structure in which the refractive index contrast is formed between air and the halide perovskite material, ${ }^{31}$ as it can be realized in $3 \mathrm{D}$ inverse opal structures. An inverse opal consists of a 3D face centered cubic array of spheres with a low refractive index (air), which are surrounded by a high refractive index medium (halide perovskite). ${ }^{32,33}$ Herein, we report on the fabrication and lasing properties of $3 \mathrm{D}$ halide perovskite DFB inverse opal structures via a facile and all-solution based colloidal crystal templating approach.

\section{RESULTS AND DISCUSSION}

3D inverse opal halide perovskite structures were prepared by an improved protocol previously developed by our group. ${ }^{32}$ The procedure involves infiltrating polystyrene opal thin films with a perovskite precursor solution followed by solvent removal to crystallize the halide perovskite material around the polystyrene and subsequent template removal by toluene at room temperature. To prepare the polystyrene opal templates, first two aqueous suspensions of monodisperse polystyrene spheres with different diameters were prepared by a modified surfactant assisted polymerization reported previously. ${ }^{32}$ The polystyrene spheres have diameters of 210 and $320 \mathrm{~nm}$ and are named "S-PS" (small-PS) and "L-PS" (large-PS), respectively.

For the application of $3 \mathrm{D}$ inverse opal structures as lasers, a high degree of ordering is the major prerequisite, as structural defects will reduce the quality of the cavity by introducing loss channels into the structure. As a consequence, high requirements have to be met for the polystyrene opal template. Important parameters that determine the suitability of the polystyrene suspensions to form high quality opal films are the monodispersity of the polystyrene particle diameter and the zeta-potential of the polystyrene spheres in the solvent. ${ }^{34,35}$ If the polystyrene particle size distribution is too polydisperse, disorder in the form of vacancies or local lattice distortions will be introduced into the opal structure, which prevents the formation of a pronounced photonic stop-band. ${ }^{35,36}$ As dynamic light scattering measurements show, the prepared polystyrene suspensions have very low polydispersity indices of 0.02 (S-PS) and 0.04 (L-PS), meaning that the particles have a very narrow particle size distribution (Table S1). Furthermore, high (according to magnitude) zeta-potentials result in stronger repulsive forces between the polystyrene particles as the distance between the particles decreases upon solvent removal. Thus, high zeta-potentials prevent the aggregation of the polystyrene particles in solution, which would prohibit the evolution of a highly ordered structure. ${ }^{34,37}$ The prepared polystyrene suspensions possess high zeta-potentials of -58 and $-50 \mathrm{mV}$, respectively. Consequently, the polystyrene particles are very stable in the suspension, which prevents their aggregation and results in well-ordered opal films upon solvent removal. The properties of the prepared polystyrene suspensions are summarized in Table S1.

At this point it should be mentioned that the inverse opal halide perovskite thin films that our group previously reported $^{32}$ did not show any lasing behavior. Only after significant improvement of the inverse opal halide perovskite structure, based on improved methods for the synthesis of the polystyrene opal templates, was the quality of the halide perovskite inverse opal samples sufficient to observe lasing. To improve the template quality, it was crucial to improve the monodispersity and the zeta-potential of the polystyrene spheres, which was achieved by modifying the ratios between styrene, sodium dodecyl sulfate and sodium persulfate and by using milli-Q water as the reaction medium during all synthesis steps. Furthermore, it was crucial to avoid convection of air during the self-assembly of the polystyrene spheres while the solvent was removed.

To prepare thin film polystyrene opals, a given amount of the prepared polystyrene suspensions was drop cast onto clean glass coverslips followed by evaporation of the water at $60{ }^{\circ} \mathrm{C}$. After approximately $10 \mathrm{~min}$, the water was completely evaporated, and strongly iridescent polystyrene films with a thickness of approximately $4 \mu \mathrm{m}$ were obtained. The prepared polystyrene opal films possess pronounced photonic stop-bands at $500 \mathrm{~nm}$ (S-PS) and $750 \mathrm{~nm}$ (L-PS), respectively (Figure 1a). The stop-band positions are (according to eq 1) in excellent agreement with the polystyrene diameters obtained from SEM measurements (Table S1).

Comparison of the relative widths $\left(\Delta \lambda / \lambda_{\max }\right)$ of the photonic stop-bands of $7.2 \%$ (S-PS) and $7.6 \%$ (L-PS) and the transmissions at the stop-band maxima of $8 \%$ (S-PS) and $17 \%$ (L-PS) indicate the high quality (good ordering) of both
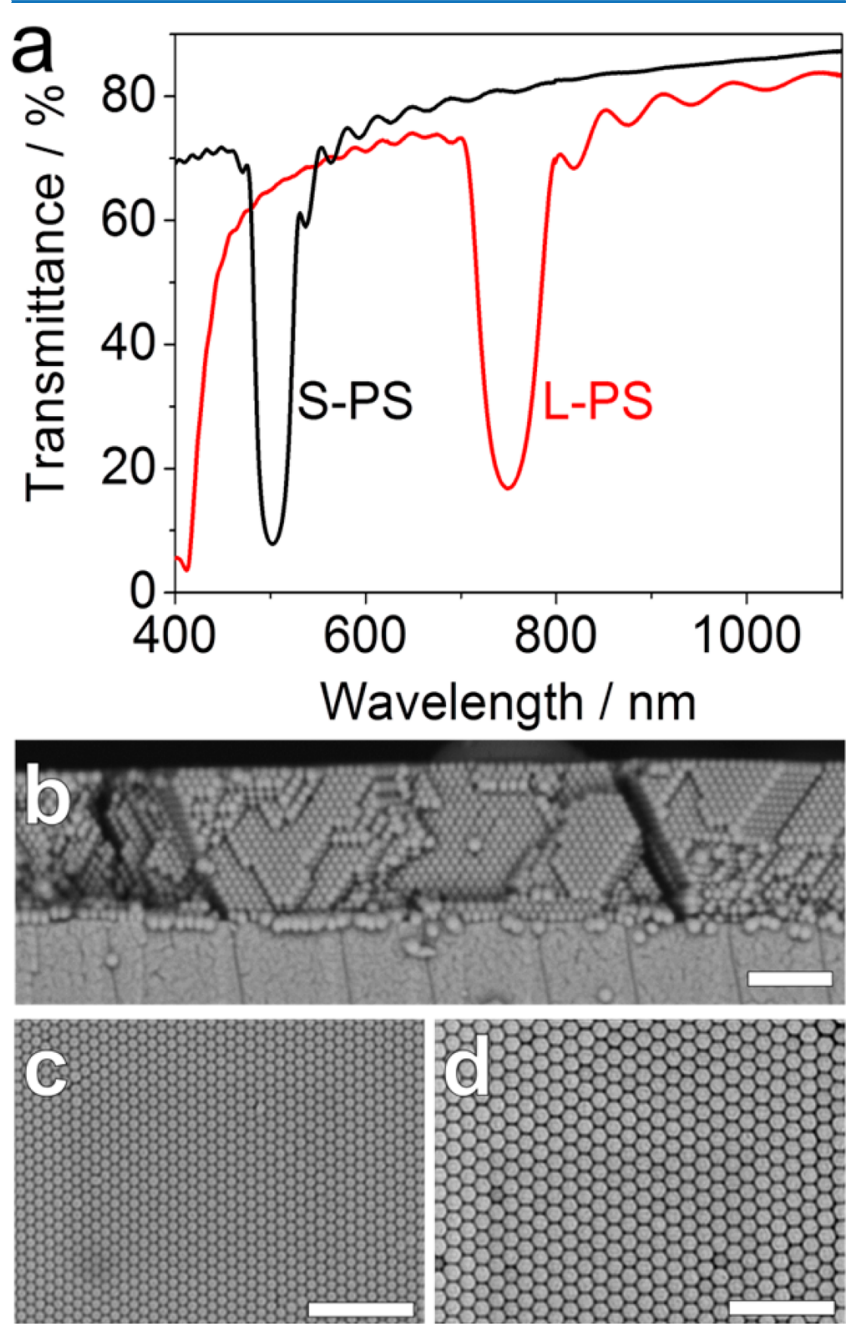

Figure 1. (a) Vis-NIR transmittance spectra of S-PS and L-PS. (b) Cross-sectional SEM image of S-PS. SEM images of (c) S-PS and (d) L-PS polystyrene opal films. The length of the scale bars is $2 \mu \mathrm{m}$. 
opal templates and show significant improvement over the structures previously reported. ${ }^{32}$ However, in comparison with L-PS, S-PS has better quality, as the relative widths and the transmission at the stop-band maxima show. Furthermore, Fabry-Perot fringes are visible in both spectra, which indicate a constant thickness of the polystyrene template of 4.0 and 4.2 $\mu \mathrm{m}$, respectively, over the illuminated area of approximately 30 $\mathrm{mm}^{2}$. The thickness obtained from the visible and near-infrared (vis-NIR) spectra is in excellent agreement with the expected value of $4.1 \mu \mathrm{m}$ and with the thickness observed from crosssectional SEM micrographs (Figure 1b). SEM micrographs of the surfaces of S-PS and L-PS further confirm the highly ordered arrangement of the polystyrene spheres in the prepared films (Figure 1c,d).

Subsequently, the prepared polystyrene opal templates were infiltrated with a $1.0 \mathrm{M} \mathrm{CH} \mathrm{CH}_{3} \mathrm{PbBr}_{3}$, or a $0.9 \mathrm{M}$ $\mathrm{CH}_{3} \mathrm{NH}_{3} \mathrm{PbI}_{3}$ dimethyl sulfoxide solution via spin coating. DMSO was selected as a solvent because of the good solubility of the halide perovskite precursors and the good stability of polystyrene in this solvent at temperatures up to $60{ }^{\circ} \mathrm{C}$. ${ }^{33}$ Subsequently, the solvent was removed by heating the samples to $60{ }^{\circ} \mathrm{C}$ (under vacuum in the case of $\mathrm{CH}_{3} \mathrm{NH}_{3} \mathrm{PbI}_{3}$ ), which leads to the crystallization of the halide perovskite material around the polystyrene spheres within approximately $10 \mathrm{~min}$. To obtain the 3D halide perovskite inverse opal thin films, the polystyrene template was selectively removed by immersing the polystyrene/halide perovskite composite film into toluene for $10 \mathrm{~s}$ as the last step of the synthesis. By this method $\mathrm{CH}_{3} \mathrm{NH}_{3} \mathrm{PbBr}_{3}$ and $\mathrm{CH}_{3} \mathrm{NH}_{3} \mathrm{PbI}_{3}$ inverse opals were prepared from S-PS and L-PS. The halide perovskite inverse opals are labeled as S-Br, L-Br, S-I, and L-I according to the employed polystyrene template and the halide component of the halide perovskite material. SEM micrographs of the prepared halide perovskite inverse opal samples are given in Figure 2 and show well replicated opal structures for $\mathrm{S}-\mathrm{Br}$ and $\mathrm{L}-\mathrm{Br}$. It should be noted that, from the SEM images, $\mathrm{S}-\mathrm{Br}$ and $\mathrm{L}-\mathrm{Br}$ seem to have a better quality than their iodide counterparts. Furthermore, the cross-sectional SEM micrograph of S-Br (Figure 2e) and the IR spectra in Figure $S 1$ prove that essentially all polystyrene is removed from the sample, also in the deepest layers of the inverse opal structure, which indicates the complete removal of the polystyrene template while maintaining a highly ordered $\mathrm{CH}_{3} \mathrm{NH}_{3} \mathrm{PbBr}_{3}$ inverse opal structure. It should be noted that the cross-sectional SEM image was recorded by breaking the inverse opal film in the center of the substrate, rather than at the edge of the prepared inverse opal film.

Figure 3 shows the diffuse reflectance vis-NIR spectra of the prepared samples. The position of the photonic stop-band for $\mathrm{S}-\mathrm{Br}$ and $\mathrm{S}-\mathrm{I}$ is at approximately $545 \mathrm{~nm}$, whereas the photonic stop-band is located at approximately $820 \mathrm{~nm}$ in the case of L$\mathrm{Br}$ and L-I. Thus, the spectral position of the photonic stopband and the optical bandgap (and therefore that of the photoluminescence) of the perovskites match in the cases of $S$ $\mathrm{Br}$ and L-I. On the other hand, no spectral overlap between the photonic stop-band and the optical bandgap is given in the samples L-Br and S-I. Consequently, anisotropic propagation of the photoluminescence, which is required for DFB lasing is expected for S-Br and L-I only. The samples L-Br and S-I will serve as control experiments since no lasing is expected from these structures.

Lasing experiments were performed with a $532 \mathrm{~nm}$ pulsed laser operated at $2000 \mathrm{~Hz}$ and $0.5 \mathrm{~ns}$ pulses. The collimated excitation beam has a diameter of $25 \mu \mathrm{m}$. Figure 4a shows the
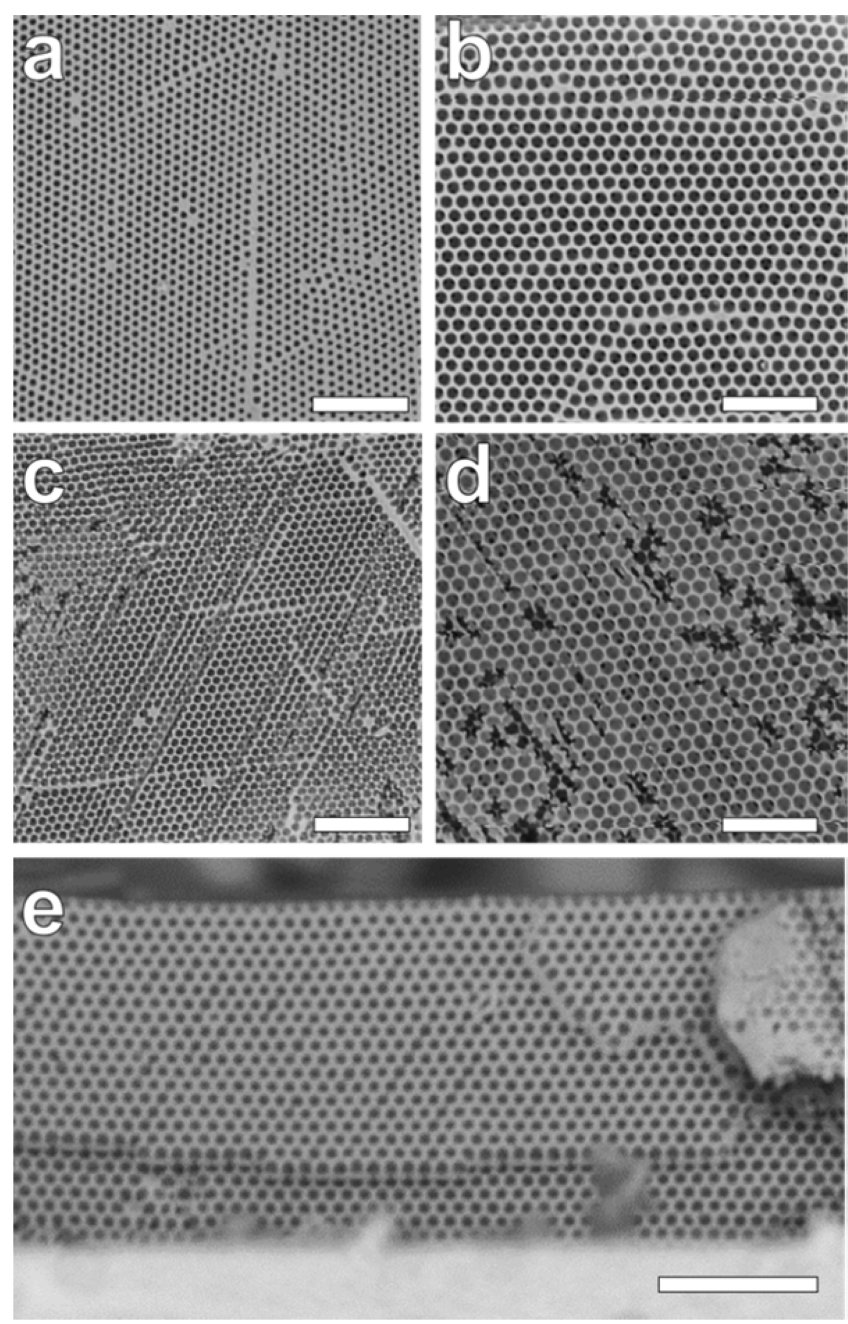

Figure 2. SEM images of halide perovskite inverse opals $\mathrm{S}-\mathrm{Br}(\mathrm{a}), \mathrm{L}-\mathrm{Br}$ (b), S-I (c), and L-I (d). Cross-sectional SEM of S-Br (e). Scale bar in each image is $2 \mu \mathrm{m}$.

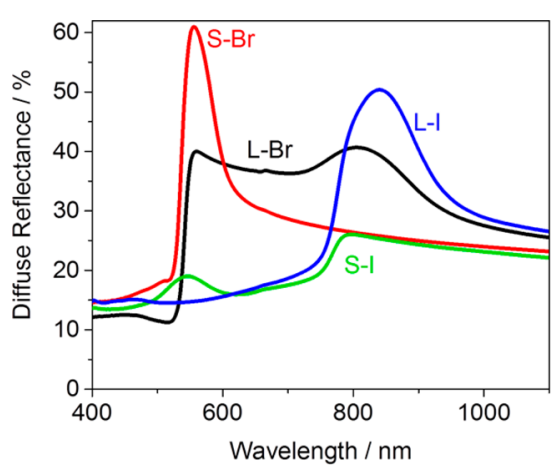

Figure 3. Vis-NIR diffuse reflectance spectra of S-Br, L-Br, S-I, and L-I.

emission intensity and the full width half-maximum (fwhm) of the emission peak of S-Br. The abrupt lasing threshold is situated at an incident energy density of approximately $1.6 \mathrm{~mJ}$ $\mathrm{cm}^{-2}$, which is higher than that of reported $1 \mathrm{D}$ and $2 \mathrm{D}$ halide perovskite DFB structures. This can be a result of the lower effective gain resulting from the low perovskite fraction in the inverse opal structure, or result from increased scattering due to irregularities of the all-solution processed 3D photonic crystal structure compared to that of sophisticated lithography techniques used to produce $1 \mathrm{D}$ and $2 \mathrm{D}$ photonic crystal 


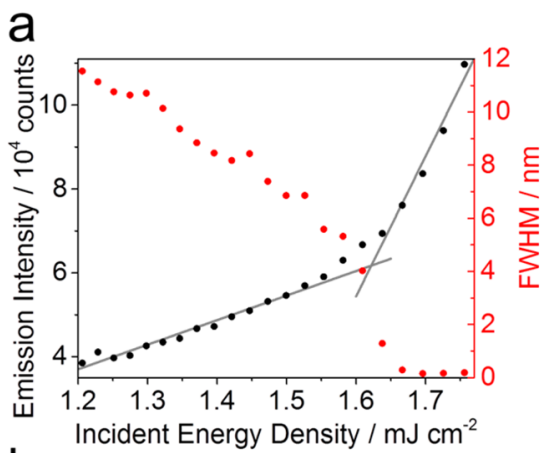

b
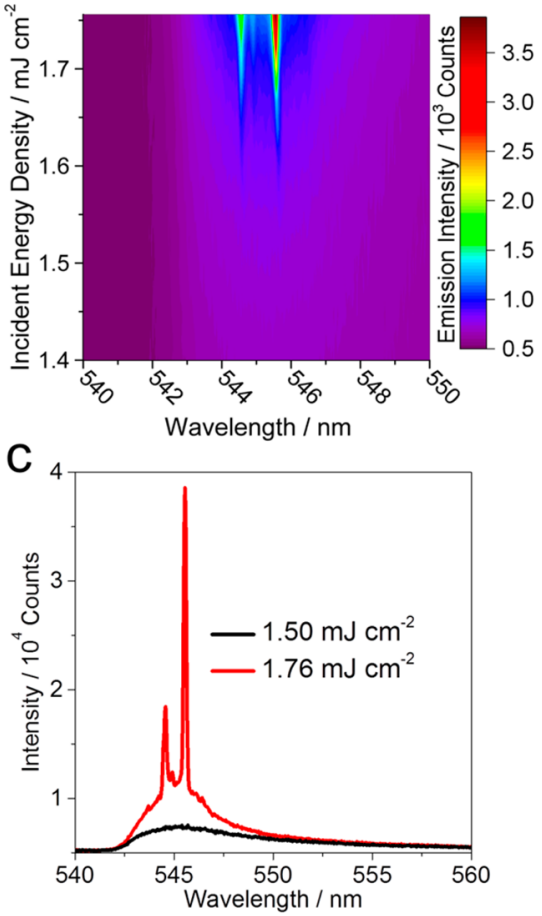

Figure 4. Emission intensity and fwhm of the photoluminescence peak of $\mathrm{S}-\mathrm{Br}$ as a function of the incident energy density (a). Emission map of $\mathrm{S}-\mathrm{Br}$ as a function of the incident energy density (b). Emission spectra at incident energy densities of $1.50 \mathrm{~mJ} \mathrm{~cm}^{-2}$ and $1.76 \mathrm{~mJ} \mathrm{~cm}^{-2}$, respectively (c).

structures. Also the high surface area of the inverse opal structure may facilitate nonradiative recombination as a result of surface defects, which could be reduced by surface passivation. $^{31}$ Since the inverse opal structures are prepared via a solution-based process, bulk trap states may serve as a nonradiative recombination pathway to increase the lasing threshold. Furthermore, the long pulse durations of 0.5 ns used in our experiments can be a cause for the relatively high lasing thresholds. It is reported that, due to the lower generated carrier density, long pump durations (nanoseconds) increase the lasing thresholds by 2 orders of magnitude as compared to short excitation pulses (femtoseconds). ${ }^{27,38}$ However, above the lasing threshold, the line width (fwhm) collapses down to $0.15 \mathrm{~nm}$ (spectral resolution of the spectrometer $0.037 \mathrm{~nm}$ ), which is among the smallest line widths as compared to previously reported DFB perovskite lasers, suggesting that the cavity quality factor is not the origin of the high lasing threshold.

Figure 4b,c shows two narrow emission lines at 544.56 and $545.54 \mathrm{~nm}$, which overlap well with the photonic stop-band's position. In theory, two different lasing mechanisms can occur in DFB structures, namely gain-coupled and index-coupled lasing. In gain-coupled lasing, a single peak at the photonic stop-band maximum is expected, whereas two modes offset from the stop-band maximum are expected for index-coupled oscillations. ${ }^{39}$ However, in real systems, spatial inhomogeneity and inhomogeneous broadening of optical transitions, among other effects, can lead to multimodal lasing, ${ }^{40}$ as it is frequently observed for $1 \mathrm{D}$ and $2 \mathrm{D}$ halide perovskite DFB structures, ${ }^{26,27}$ as well as for DFB structures made from other materials. ${ }^{41-44}$ In the 3D halide perovskite inverse opal structure, inhomogeneity of the DFB structure within the illuminated area and inhomogeneous broadening of the material's optical transition from local variations (defects, strain, etc.) can lead to nonuniform saturation behaviors in different parts of the illuminated area, which allows multimodal lasing. Random lasers are also often multimodal, but in this case random lasing is implausible for the following reasons: (1) no lasing was observed from L-Br, whose photonic stop-band does not overlap with the material's gain spectrum (Figure S2); (2) no lasing was observed from a less ordered photonic crystal structure made previously using the same sized polystyrene beads, which excludes random lasing from resonance-driven scattering; $^{45}$ (3) the lasing is stable over minutes (Figure S3), while random lasing modes often fluctuate sensitively in time. ${ }^{46}$

Table S2 summarizes the pulse durations, lasing thresholds and fwhms of this work and of previously reported perovskite lasers. It should be noted, that a $540 \mathrm{~nm}$ long-pass filter was used to prevent the excitation beam from reaching the detector, which also cuts off parts of the $\mathrm{CH}_{3} \mathrm{NH}_{3} \mathrm{PbBr}_{3}$ 's photoluminescence and results in the decreasing fwhm at increasing incident energy densities below the lasing threshold. Similar to the spatial inhomogeneity of the efficiency of halide perovskite solar cells, at some parts of the $20 \times 20 \mathrm{~mm}^{2}$ photonic crystal film no lasing was observed. In our case this can be due to disorder that occurs unavoidably at some parts of the film during the polystyrene self-assembly process. These observations demonstrate the importance of a well-defined structure, as already discussed in previous reports. ${ }^{38}$

To evaluate the stability of $\mathrm{S}-\mathrm{Br}$ under laser excitation, the sample was illuminated for $18 \mathrm{~min}$ with an incident energy density of $1.76 \mathrm{~mJ} \mathrm{~cm}^{-2}$ (which is the maximal power of the excitation beam) with a repetition rate of $2000 \mathrm{~Hz}$. Under these conditions, constant lasing emission was observed from the sample. As depicted in Figure S3, the emission spectrum and the emission intensity are constant over the observed time frame, which indicates that the 3D DFB structure does not undergo degradation from the excitation beam, despite the high lasing threshold. To exclude lasing mechanisms that do not stem from the overlap of the photonic stop-band and the optical bandgap, L-Br, which does not have an overlap between the photonic stop-band and the bandgap, was used as a reference sample. As expected, no lasing was observed for this sample, which excludes the presence of lasing mechanisms other than DFB lasing (Figure S2).

Under the same illumination conditions, no lasing was observed for L-I, although it has a photonic stop-band within the bandgap of $\mathrm{CH}_{3} \mathrm{NH}_{3} \mathrm{PbI}_{3}$ and, therefore, would be expected to lase. The absent lasing action can be explained by the lower degree of ordering of L-I, which is reflected by the SEM micrographs (Figure 2) and the less pronounced photonic stopband as compared to $\mathrm{S}-\mathrm{Br}$ (Figure 3). Another possibility for the absent lasing emission could be the lower stability of 
$\mathrm{CH}_{3} \mathrm{NH}_{3} \mathrm{PbI}_{3}$, as compared to $\mathrm{CH}_{3} \mathrm{NH}_{3} \mathrm{PbBr}_{3}$, which is wellknown in the literature. Therefore, lasing experiments were also conducted with poly(methyl methacrylate) (PMMA)-coated LI. However, still no lasing was also observed for this sample under these illumination conditions.

To verify lasing from L-I required higher excitation energy densities. These were achieved by focusing the previously collimated excitation laser beam onto the sample. As a result of the increased incident energy densities, lasing with a sharp onset and fwhm of $0.16 \mathrm{~nm}$ was also observed from L-I (Figure 5 ). However, immediate irreversible degradation of the sample

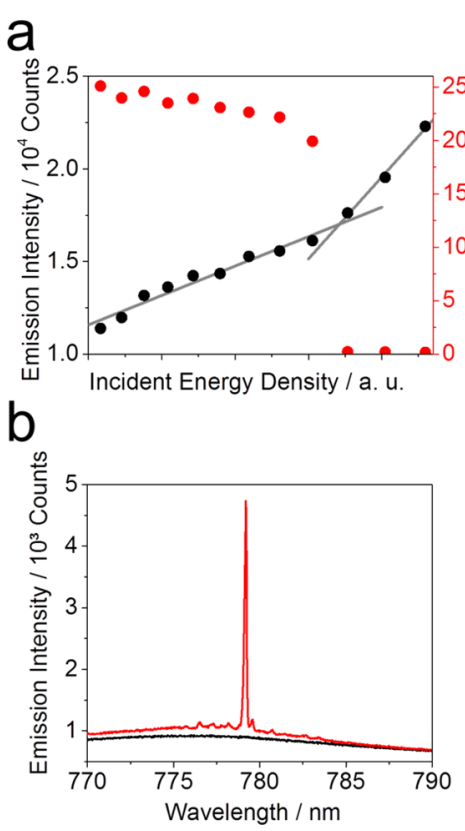

Figure 5. Emission intensity and fwhm of the photoluminescence peak of L-I as a function of the incident energy density (a). L-I emission spectrum above (red line) and below (black line) the lasing threshold.

at the center of the excitation beam was observed after a single shot with maximal incident energy density (Figure S4). Thus, it is reasonable to assume that the lasing emission originates from excitation of the higher order maxima of the Airy disc pattern of the excitation beam. Since evidence for this assumption cannot be given, we prefer not to give values for the excitation energy densities for the experiments performed with a focused laser beam. The degradation of the sample by the focused laser beam was also confirmed by long-term stability measurements which show a $20 \%$ decrease of the emission intensity within the first 2 min of excitation and unsteady emission spectra over the observed time frame (Figure S5). This implies that, besides the very fast (single pulse) degradation of the $\mathrm{CH}_{3} \mathrm{NH}_{3} \mathrm{PbI}_{3}$, another slower degradation mechanism is present as well. This can either be thermal degradation or light-induced degradation in the presence of oxygen to $\mathrm{PbI}_{2}$, as previously observed in $\mathrm{CH}_{3} \mathrm{NH}_{3} \mathrm{PbI}_{3}$ solar cells. ${ }^{47}$

\section{CONCLUSION}

In conclusion, we have demonstrated the first all-solution based protocol for the fabrication of $\mathrm{CH}_{3} \mathrm{NH}_{3} \mathrm{PbBr}_{3}$ and $\mathrm{CH}_{3} \mathrm{NH}_{3} \mathrm{PbI}_{3}$ distributed feedback lasers based on a $3 \mathrm{D}$ photonic crystal nanostructure. By this method distributed feedback halide perovskite lasers were prepared without expensive and elaborate lithography techniques that are required by the existing protocols to prepare nanostructured halide perovskite materials and devices. The prepared $\mathrm{CH}_{3} \mathrm{NH}_{3} \mathrm{PbBr}_{3}$ 3D DFB laser shows narrow lasing emission with a fwhm as low as $0.15 \mathrm{~nm}$ and good long-term stability under pulsed laser excitation above the lasing threshold of 1.6 $\mathrm{mJ} \mathrm{cm} \mathrm{cm}^{-2}$ in ambient atmosphere. Our results open a novel path toward the low cost and large scale fabrication of hybrid halide perovskite lasers.

\section{METHODS}

Polystyrene Template. Prior to use, all glassware was extensively cleaned with piranha solution (3:1 concn $\mathrm{H}_{2} \mathrm{SO}_{4}$ / $30 \% \mathrm{H}_{2} \mathrm{O}_{2}$ ), followed by rinsing with milli-Q water, to allow the fabrication of high quality polystyrene spheres. Monodispersed polystyrene suspensions with two different polystyrene diameters were prepared via a surfactant-assisted emulsion polymerization modified from existing literature. ${ }^{32}$ First, $50 \mathrm{~mL}$ of milli-Q water was heated to $80{ }^{\circ} \mathrm{C}$ in a $250 \mathrm{~mL}$ three-neck round-bottom flask equipped with a reflux condenser and an $\mathrm{Ar}$ inlet under a constant Ar flow for $60 \mathrm{~min}$ to remove dissolved oxygen from the water. Subsequently, polystyrene spheres with a diameter of $210 \mathrm{~nm}(320 \mathrm{~nm})$ were prepared by adding 7.5 $\mathrm{mL}(11 \mathrm{~mL})$ of styrene (previously extracted $3 \times$ with $1 \mathrm{M}$ $\mathrm{NaOH}$ and $3 \times$ with deionized water) to the milli-Q water. After the mixture reached a stable temperature of $80{ }^{\circ} \mathrm{C}, 216.7$ $\mathrm{mg}(154.8 \mathrm{mg})$ sodium persulfate and $32.2 \mathrm{mg}$ sodium dodecyl sulfate dissolved in $10 \mathrm{~mL}$ of milli-Q water were rapidly added to the mixture under an Ar counterflow. The mixture was stirred for $5 \mathrm{~h}$ under an Ar atmosphere. Subsequently, the white suspension was sedimented by centrifugation (30 min, 16500 $\mathrm{rpm}$ ). The supernatant liquid was removed and the polystyrene spheres were redispersed in milli- $Q$ water via sonication. This process was repeated three times. Finally, the suspensions were diluted with milli-Q water to 0.5 wt \% polystyrene. Polystyrene opal films were prepared on $20 \times 20 \mathrm{~mm}^{2}$ glass substrates previously cleaned by sonication in acetone, isopropanol and milli-Q water for $20 \mathrm{~min}$ each. Hydrophilicity was achieved by immersing the clean glass slides in a freshly prepared piranha solution. Subsequently, the glass slides were placed on a precisely vertically aligned hot plate. To obtain high quality polystyrene opal films, it was found crucial that convection of air around the sample during solvent evaporation was minimized. For this reason, a cylinder was placed on the hot plate around the sample to reduce air convection. Subsequently, $235 \mu \mathrm{L}$ of a $0.5 \%$ polystyrene solution was dropped on the glass slide and the water was slowly evaporated at $60{ }^{\circ} \mathrm{C}$ to give an average thickness of the polystyrene opal film of $4.1 \mu \mathrm{m}$. Evaporation of the solvent leads to the self-assembly of the polystyrene spheres in a close-packed array with the (111) surface parallel to the glass surface.

$\mathrm{CH}_{3} \mathrm{NH}_{3} \mathrm{PbBr}_{3}$ and $\mathrm{CH}_{3} \mathrm{NH}_{3} \mathrm{Pbl}_{3}$ Inverse Opal Films. The preparation of the inverse opal perovskite films followed the procedure previously reported. ${ }^{32} \mathrm{CH}_{3} \mathrm{NH}_{3} \mathrm{PbBr}_{3}$ inverse opals were prepared by dropping $150 \mu \mathrm{L}$ of a solution consisting of 1 $\mathrm{M}$ methylammonium bromide and $1 \mathrm{M}$ lead(II) bromide in DMSO on the polystyrene opal template and spin-casting at $2000 \mathrm{rpm}$ for $10 \mathrm{~s}$. $\mathrm{MAPbI}_{3}$ inverse opals were prepared similarly with the exception that a $0.9 \mathrm{M} \mathrm{CH}_{3} \mathrm{NH}_{3} \mathrm{PbI}_{3}$ solution in DMSO was applied to avoid formation of bulk crystals on the surface of the inverse opal structure. After drying at $60{ }^{\circ} \mathrm{C}$ for $1 \mathrm{~h}$ (under vacuum in the case of $\mathrm{CH}_{3} \mathrm{NH}_{3} \mathrm{PbI}_{3}$ ), the polystyrene template was removed by immersing the film for 10 $s$ in toluene at room temperature. 
Sample Characterization. Transmission vis-NIR spectra were recorded with a Varian Cary 4000 spectrometer equipped with self-built sample holder. Vis-NIR diffuse reflectance spectra were recorded with a PerkinElmer Lambda 365 spectrophotometer equipped with an integrating sphere. Samples for scanning electron microscopy (SEM) were prepared by sputter coating a $10 \mathrm{~nm}$ Au layer on the samples. SEM micrographs were recorded with a Hitachi TM3030 with an acceleration voltage of $15 \mathrm{kV}$ in back scattered electron contrast. Dynamic light scattering experiments for particle size distribution and $\xi$-potential determination were carried out on a Malvern Nano-ZS Zetasizer with a $633 \mathrm{~nm}$ laser. The detector angle was $173^{\circ}$ in each measurement. IR spectra were obtained from powders by scratching off the polystyrene, polystyrene/ $\mathrm{CH}_{3} \mathrm{NH}_{3} \mathrm{PbBr}_{3}$, and $\mathrm{CH}_{3} \mathrm{NH}_{3} \mathrm{PbBr}_{3}$ films, respectively. The spectrometer was a Nicolet MAGNA-IR 560 equipped with single reflection diamond ATR cell.

Lasing Experiments. All lasing experiments were performed in ambient air. The setup is essentially an inverted fluorescence microscope equipped with real-space and backfocal plane (Fourier-space) imaging, as well as spectral (Fourier) imaging as described by Koenderink et al. ${ }^{48}$ Samples are placed on the platform of the inverted microscope with the substrate facing the objective (Nikon Plan Fluor, air, 100×, NA $=0.9$ ). The samples are pumped through the objective and substrate side with collimated laser pulses of $532 \mathrm{~nm}$ wavelength (Teem Photonics, type STG-03E-1S0), with a beam diameter at the sample of about $25 \mu \mathrm{m}$ adjusted by an iris. For focused illumination in the plane of the sample, the collimated laser beam was incident on the back focal plane of the objective directly. Each pulse has a width of $0.5 \mathrm{~ns}$. The laser power is controlled by a computer-controlled acoustooptic modulator. The emission is passed through a long pass filters to remove pump laser light, and is imaged on either a $\mathrm{Si}$ CCD camera or a Shamrock303i spectrometer with an Si CCD detector (spectral resolution of $0.037 \mathrm{~nm}$ ), through the same objective and beam splitter, and a $1 \times$ telescope and a tube lens. In addition to collecting images and spectra in real space, Fourier images are obtained by adding a lens on a flip mount at a focal distance from the back-focal plane of the objective.

\section{ASSOCIATED CONTENT}

\section{S Supporting Information}

The Supporting Information is available free of charge on the ACS Publications website at DOI: 10.1021/acsphotonics.7b00780.

Polystyrene diameters measured by various methods, polydispersities and zeta-potentials of polystyrene suspensions, overview of pump durations, lasing thresholds, and fwhms of previously reported halide perovskite DFB structures, ATR-FT-IR spectra of polystyrene template, $\mathrm{CH}_{3} \mathrm{NH}_{3} \mathrm{PbBr}_{3}$ /polystyrene composite, and of inverse opal $\mathrm{CH}_{3} \mathrm{NH}_{3} \mathrm{PbBr}_{3}$ after template removal, emission spectra and normalized emission intensity of $\mathrm{S}-\mathrm{Br}$ under long time excitation, emission intensities and fwhm of $\mathrm{L}-\mathrm{Br}$ at various excitation energy densities, optical microscope image of L-I after illumination with focused beam, emission spectra, and normalized emission intensity of L-I under long time excitation (PDF).

\section{AUTHOR INFORMATION}

\section{Corresponding Author}

*E-mail: tueysuez@kofo.mpg.de.

ORCID $\odot$

Kun Chen: 0000-0002-1403-0118

Harun Tüysüz: 0000-0001-8552-7028

\section{Author Contributions}

The manuscript was written through contributions of all authors. All authors have given approval to the final version of the manuscript.

Notes

The authors declare no competing financial interest.

\section{ACKNOWLEDGMENTS}

This work was supported by the MAXNET Energy consortium of Max Planck Society, the Cluster of Excellence RESOLV (EXC 1069) funded by the Deutsche Forschungsgemeinschaft (DFG), and Fonds der Chemischen Industrie (FCI). The work at AMOLF is part of the research program of the "Nederlandse Organisatie voor Wetenschappelijk Onderzoek" (NWO). This work was supported by the European Research Council (Grant Agreement No. 337328) and by an industrial partnership with Philips. The authors would like to thank Prof. Femius Koenderink and Cocoa Guo for generously ceding their setup for the lasing experiments, as well as for fruitful discussions and helpful comments on the manuscript.

\section{REFERENCES}

(1) Wehrenfennig, C.; Eperon, G. E.; Johnston, M. B.; Snaith, H. J.; Herz, L. M. High Charge Carrier Mobilities and Lifetimes in Organolead Trihalide Perovskites. Adv. Mater. 2014, 26, 1584-1589.

(2) Yin, W.-J.; Shi, T.; Yan, Y. Unique Properties of Halide Perovskites as Possible Origins of the Superior Solar Cell Performance. Adv. Mater. 2014, 26, 4653-4658.

(3) Saparov, B.; Mitzi, D. B. Organic-Inorganic Perovskites: Structural Versatility for Functional Materials Design. Chem. Rev. 2016, 116, 4558-4596.

(4) Manser, J. S.; Christians, J. A.; Kamat, P. V. Intriguing Optoelectronic Properties of Metal Halide Perovskites. Chem. Rev. 2016, 116, 12956-13008.

(5) Green, M. A.; Ho-Baillie, A.; Snaith, H. J. The Emergence of Perovskite Solar Cells. Nat. Photonics 2014, 8, 506-514.

(6) Wells, H. L. Über die Cäsium- und Kalium-Bleihalogenide. $Z$. Anorg. Allg. Chem. 1893, 3, 195-210.

(7) Mitzi, D. B.; Feild, C. A.; Schlesinger, Z.; Laibowitz, R. B. Transport, Optical, and Magnetic-Properties of the Conducting Halide Perovskite $\mathrm{CH}_{3} \mathrm{NH}_{3} \mathrm{SnI}_{3}$. J. Solid State Chem. 1995, 114, 159-163.

(8) Mitzi, D. B.; Feild, C. A.; Harrison, W. T. A.; Guloy, A. M. Conducting Tin Halide with a Layered Oganic-Based Perovskite Structure. Nature 1994, 369, 467-469.

(9) Kojima, A.; Teshima, K.; Shirai, Y.; Miyasaka, T. Organometal Halide Perovskites as Visible-Light Sensitizers for Photovoltaic Cells. J. Am. Chem. Soc. 2009, 131, 6050-6051.

(10) Correa-Baena, J.-P.; Abate, A.; Saliba, M.; Tress, W.; Jacobsson, T. J.; Grätzel, M.; Hagfeldt, A. The Rapid Evolution of Highly Efficient Perovskite Solar Cells. Energy Environ. Sci. 2017, 10, 710-727.

(11) Cho, H.; Jeong, S.-H.; Park, M.-H.; Kim, Y.-H.; Wolf, C.; Lee, C.-L.; Heo, J. H.; Sadhanala, A.; Myoung, N.; Yoo, S.; Im, S. H.; Friend, R. H.; Lee, T.-W. Overcoming the Electroluminescence Efficiency Limitations of Perovskite Light-Emitting Diodes. Science 2015, 350, 1222-1225.

(12) Fu, Y.; Zhu, H.; Stoumpos, C. C.; Ding, Q.; Wang, J.; Kanatzidis, M. G.; Zhu, X.; Jin, S. Broad Wavelength Tunable Robust Lasing from Single-Crystal Nanowires of Cesium Lead Halide Perovskites $\left(\mathrm{CsPbX}_{3}, \mathrm{X}=\mathrm{Cl}, \mathrm{Br}, \mathrm{I}\right)$. ACS Nano 2016, 10, 7963-7972. 
(13) Tan, Z.-K.; Moghaddam, R. S.; Lai, M. L.; Docampo, P.; Higler, R.; Deschler, F.; Price, M.; Sadhanala, A.; Pazos, L. M.; Credgington, D.; Hanusch, F.; Bein, T.; Snaith, H. J.; Friend, R. H. Bright LightEmitting Diodes Based on Organometal Halide Perovskite. Nat. Nanotechnol. 2014, 9, 687-692.

(14) Stranks, S. D.; Snaith, H. J. Metal-Halide Perovskites for Photovoltaic and Light-Emitting Devices. Nat. Nanotechnol. 2015, 10, 391-402.

(15) Chen, S. T.; Roh, K.; Lee, J.; Chong, W. K.; Lu, Y.; Mathews, N.; Sum, T. C.; Nurmikko, A. A Photonic Crystal Laser from Solution Based Organo-Lead Iodide Perovskite Thin Films. ACS Nano 2016, 10, 3959-3967.

(16) Jia, Y.; Kerner, R. A.; Grede, A. J.; Brigeman, A. N.; Rand, B. P.; Giebink, N. C. Diode-Pumped Organo-Lead Halide Perovskite Lasing in a Metal-Clad Distributed Feedback Resonator. Nano Lett. 2016, 16, $4624-4629$.

(17) Zhang, Q.; Ha, S. T.; Liu, X.; Sum, T. C.; Xiong, Q. RoomTemperature Near-Infrared High-Q Perovskite Whispering-Gallery Planar Nano Lasers. Nano Lett. 2014, 14, 5995-6001.

(18) Xing, J.; Liu, X. F.; Zhang, Q.; Ha, S. T.; Yuan, Y. W.; Shen, C.; Sum, T. C.; Xiong, Q. Vapor Phase Synthesis of Organometal Halide Perovskite Nanowires for Tunable Room-Temperature Nanolasers. Nano Lett. 2015, 15, 4571-4577.

(19) Xing, G.; Mathews, N.; Lim, S. S.; Yantara, N.; Liu, X.; Sabba, D.; Grätzel, M.; Mhaisalkar, S.; Sum, T. C. Low-Temperature Solution-Processed Wavelength-Tunable Perovskites for Lasing. Nat. Mater. 2014, 13, 476-480.

(20) Deschler, F.; Price, M.; Pathak, S.; Klintberg, L. E.; Jarausch, D. D.; Higler, R.; Huttner, S.; Leijtens, T.; Stranks, S. D.; Snaith, H. J.; Atature, M.; Phillips, R. T.; Friend, R. H. High Photoluminescence Efficiency and Optically Pumped Lasing in Solution-Processed Mixed Halide Perovskite Semiconductors. J. Phys. Chem. Lett. 2014, 5, 14211426

(21) Perumal, P.; Wang, C. S.; Boopathi, K. M.; Haider, G.; Liao, W. C.; Chen, Y. F. Whispering Gallery Mode Lasing from Self-Assembled Hexagonal Perovskite Single Crystals and Porous Thin Films Decorated by Dielectric Spherical Resonators. ACS Photonics 2017, $4,146-155$.

(22) Liao, Q.; Hu, K.; Zhang, H.; Wang, X.; Yao, J.; Fu, H. Perovskite Microdisk Microlasers Self-Assembled from Solution. Adv. Mater. 2015, 27, 3405-3410.

(23) Wang, K. Y.; Sun, W. Z.; Li, J. K.; Gu, Z. Y.; Xiao, S. M.; Song, Q. H. Unidirectional Lasing Emissions from $\mathrm{CH}_{3} \mathrm{NH}_{3} \mathrm{PbBr}_{3}$ Perovskite Microdisks. ACS Photonics 2016, 3, 1125-1130.

(24) Park, K.; Lee, J. W.; Kim, J. D.; Han, N. S.; Jang, D. M.; Jeong, S.; Park, J.; Song, J. K. Light-Matter Interactions in Cesium Lead Halide Perovskite Nanowire Lasers. J. Phys. Chem. Lett. 2016, 7, 37033710

(25) Zhu, H.; Fu, Y.; Meng, F.; Wu, X.; Gong, Z.; Ding, Q.; Gustafsson, M. V.; Trinh, M. T.; Jin, S.; Zhu, X. Y. Lead Halide Perovskite Nanowire Lasers with Low Lasing Thresholds and High Quality Factors. Nat. Mater. 2015, 14, 636-642.

(26) Brenner, P.; Stulz, M.; Kapp, D.; Abzieher, T.; Paetzold, U. W.; Quintilla, A.; Howard, I. A.; Kalt, H.; Lemmer, U. Highly Stable Solution Orocessed Metal-Halide Perovskite Lasers on Nanoimprinted Distributed Feedback Structures. Appl. Phys. Lett. 2016, 109, 141106.

(27) Whitworth, G. L.; Harwell, J. R.; Miller, D. N.; Hedley, G. J.; Zhang, W.; Snaith, H. J.; Turnbull, G. A.; Samuel, I. D. W. Nanoimprinted distributed feedback lasers of solution processed hybrid perovskites. Opt. Express 2016, 24, 23677-23684.

(28) Xing, G.; Kumar, M. H.; Chong, W. K.; Liu, X.; Cai, Y.; Ding, H.; Asta, M.; Grätzel, M.; Mhaisalkar, S.; Mathews, N.; Sum, T. C. Solution-Processed Tin-Based Perovskite for Near-Infrared Lasing. Adv. Mater. 2016, 28, 8191-8196.

(29) Kogelnik, H.; Shank, C. V. Stimulated Emission in a Periodic Structure. Appl. Phys. Lett. 1971, 18, 152-154.

(30) Schroden, R. C.; Al-Daous, M.; Blanford, C. F.; Stein, A. Optical Properties of Inverse Opal Photonic Crystals. Chem. Mater. 2002, 14, $3305-3315$
(31) Pourdavoud, N.; Wang, S.; Mayer, A.; Hu, T.; Chen, Y. W.; Marianovich, A.; Kowalsky, W.; Heiderhoff, R.; Scheer, H. C.; Riedl, T. Photonic Nanostructures Patterned by Thermal Nanoimprint Directly into Organo- Metal Halide Perovskites. Adv. Mater. 2017, 29, 1605003 .

(32) Schünemann, S.; Chen, K.; Brittman, S.; Garnett, E.; Tüysüz, H. Preparation of Organometal Halide Perovskite Photonic Crystal Films for Potential Optoelectronic Applications. ACS Appl. Mater. Interfaces 2016, 8, 25489-25495.

(33) Chen, K.; Tüysüz, H. Morphology-Controlled Synthesis of Organometal Halide Perovskite Inverse Opals. Angew. Chem., Int. Ed. 2015, 54, 13806-13810.

(34) Han, M. G.; Shin, C. G.; Jeon, S. J.; Shim, H.; Heo, C. J.; Jin, H.; Kim, J. W.; Lee, S. Full Color Tunable Photonic Crystal from Crystalline Colloidal Arrays with an Engineered Photonic Stop-Band. Adv. Mater. 2012, 24, 6438-6444.

(35) Marlow, F.; Muldarisnur; Sharifi, P.; Brinkmann, R.; Mendive, C. Opals: Status and Prospects. Angew. Chem., Int. Ed. 2009, 48, 62126233.

(36) Rengarajan, R.; Mittleman, D.; Rich, C.; Colvin, V. Effect of Disorder on the Optical Properties of Colloidal Crystals. Phys. Rev. E 2005, 71, 016615.

(37) Liu, L.-y.; Wang, X.-f.; Cheng, B.; Zhang, C.-x. Modification of Spherical $\mathrm{SiO}_{2}$ Particles via Electrolyte for High Zeta Potential and Self-Assembly of $\mathrm{SiO}_{2}$ Photonic Crystal. J. Braz. Chem. Soc. 2009, 20, $46-50$.

(38) Kawabe, Y.; Spiegelberg, C.; Schulzgen, A.; Nabor, M. F.; Kippelen, B.; Mash, E. A.; Allemand, P. M.; Kuwata-Gonokami, M.; Takeda, K.; Peyghambarian, N. Whispering-Gallery-Mode Microring Laser using a Conjugated Polymer. Appl. Phys. Lett. 1998, 72, 141143.

(39) Misawa, H.; Nishijima, Y.; Ueno, K.; Juodkazis, S.; Mizeikis, V.; Maeda, M.; Minaki, M. Tunable Single-mode Photonic Lasing from Zirconia Inverse Opal Photonic Crystals. Opt. Express 2008, 16, 13676-13684.

(40) Siegman, A. E. In Lasers; Kelly, A., Ed.; University Science Books: Sausalitos, CA, 1986; pp 462-466.

(41) Tandaechanurat, A.; Ishida, S.; Guimard, D.; Nomura, M.; Iwamoto, S.; Arakawa, Y. Lasing oscillation in a three-dimensional photonic crystal nanocavity with a complete bandgap. Nat. Photonics 2011, 5, 91-94.

(42) Nishijima, Y.; Joudkazis, S. Optical Characterization and Lasing in Three-dimensional Opal-structures. Front. Mater. 2015, 2, 1-10.

(43) Mekis, A.; Meier, M.; Dodabalapur, A.; Slusher, R. E.; Joannopoulos, J. D. Lasing Mechanism in Two-dimensional Photonic Crystal Lasers. Appl. Phys. A: Mater. Sci. Process. 1999, 69, 111-114.

(44) Nishijima, Y.; Ueno, K.; Juodkazis, S.; Mizeikis, V.; Fujiwara, H.; Sasaki, K.; Misawa, H. Lasing with Well-defined Cavity Modes in Dyeinfiltrated Silica Inverse Opals. Opt. Express 2009, 17, 2976-2983.

(45) Gottardo, S.; Sapienza, R.; Garcia, P. D.; Blanco, A.; Wiersma, D. S.; Lopez, C. Resonance-driven random lasing. Nat. Photonics 2008, 2, 429-432.

(46) González, I. R. R.; Lima, B. C.; Pincheira, P. I. R.; Brum, A. A.; Macêdo, A. M. S.; Vasconcelos, G. L.; de S. Menezes, L.; Raposo, E. P.; Gomes, A. S. L.; Kashyap, R. Turbulence Hierarchy in a Random Fibre Laser. Nat. Commun. 2017, 8, 15731.

(47) Abdelmageed, G.; Jewell, L.; Hellier, K.; Seymour, L.; Luo, B. B.; Bridges, F.; Zhang, J. Z.; Carter, S. Mechanisms for Light Induced Degradation in $\mathrm{MAPbI}_{3}$ Perovskite Thin Films and Solar Cells. Appl. Phys. Lett. 2016, 109, 233905.

(48) Schokker, A. H.; Koenderink, A. F. Lasing at the Band Edges of Plasmonic Lattices. Phys. Rev. B: Condens. Matter Mater. Phys. 2014, 90, 155452 . 